\title{
A Novel System of Fingerprint Recognition Approach for Immigration Control
}

\author{
Fatai .O. Waheed ${ }^{1}$, Awotunde .J. Bamidele ${ }^{2}$, Matiluko .E. Opeyemi ${ }^{3}$ \\ ${ }^{1,2}$ Computer Science Department, Faculty of Information and Communication Science, University of Ilorin, \\ Ilorin, Nigeria \\ ${ }^{3}$ Center for Systems and Information Services, Landmark University, Omu-Aran, Nigeria
}

\begin{abstract}
Insecurity is a pressing and important issue that needs urgent attention in Nigeria. The dramatic increase in worldwide flow of people, goods, and ideas has created a new group of threats. These are global organized crimes such as smuggling, trafficking, terrorism; cross-border crimes, illegal migration, amongst others. It has been noted that these foreigners device a means of beating security devices put in place at the border. The Nigerian Immigration uses Automated Fingerprint identification system (AFIS) minutiae base, which is the most popular and extensively used method for fingerprint identification. It show poor performance for real time authentication and it has less noise tolerant unlike the correlation base approach. The authors therefore proposes an hybrid fingerprinting method that combines two approaches namely minutiae and correlation methods that can help identify identity fraud. The sole idea behind this is that one technique makes up for the deficiency of the other by so doing we obtain a reliable and efficient result and provide a more secured environment where immigrants will not be able to beat the system with their fraudulent tactics or acts as currently experienced. Java programming language is used because of its platform independence, scalability, easy integration, implementation and upgrade.
\end{abstract}

Keywords: Security, Immigration, Control, Identity fraud, Fingerprint, Minutiae.

\section{Introduction}

Fingerprint has been one of the core methodologies for identification of persons. It remains a reliable, efficient and commonly accepted biometric. Fingerprint databases are in use worldwide for the purposes of personal identification, border control as well as to facilitate criminal forensic investigation. Many countries have multiple fingerprint databases, with each database serving a specific purpose and sometimes they complement each others.

It is established that terrorist activities have negative effect on National development in Nigeria [1]. The Immigrant seen as the real threats are those who cross the borders without valid documents. Recent development in the security situation in Nigeria shows that terrorist groups are predominantly foreigners from neighbouring countries. This development poses security threats due to poor border control. Nigerian Immigration Service uses AFIS which is minutiae based and it's less noise tolerant thereby producing a blur images in some cases.

Minutiae-based methods are the most popular ones being included in almost all contemporary fingerprint identification and verification systems [2]. This method represents the fingerprint by its local features like, terminations and bifurcations called as minutia. Minutiae are small points of interest in the fingerprint image. The minutiae-based method does the recognition in two stages that is minutiae extraction and minutiae matching [3]. But the cross correlation based technique is a promising approach to fingerprint authentication for the new generation of high resolution and touch less fingerprint sensors.

The authors provided a fingerprinting method which combines two approaches namely minutia and correlation approaches for the process of extraction and authentication, the idea is to see how the shortcomings of one can make up for the other. It also follows that efforts should be made to reduce terrorist activities by Government through appropriate sanction and machines (fingerprint device) put in place at the borders i.e. tackling of inherent challenges through integration of biometric-based technology into Nigeria immigration system.

In this paper the authors will examine the features of both the minutia and correlation approaches for the process of extraction and authentication and see how both methods can complement each others.

\section{Related Works}

Nigeria could be said to be much more vulnerable to the threats than any country in Africa. Being the largest population and market in Africa (over 140 million people), peoples of different backgrounds enter the country for socio-economic purposes from all over the world [4]. Almost all the migration and security stakeholders seemed to agree that its borders are leaky and poorly monitored. Also almost on the daily basis 
would one read or hear of migration-related offences committed by Nigerians and non-Nigerians within and outside its borders respectively [4].

A fingerprint is a pattern of ridges and furrows located on the tip of each finger. Fingerprints were used for personal identification for many centuries and the matching accuracy was very high Home Affairs Justice (2006). Patterns have been extracted by creating an inked impression of the fingertip on paper. Today, compact sensors provide digital images of these patterns. Fingerprint recognition for identification acquires the initial image through live scan of the finger by direct contact with a reader device that can also check for validating attributes such as temperature and pulse. In real-time verification systems, images acquired by sensors are used by the feature extraction module to compute the feature values. The feature values typically correspond to the position and orientation of certain critical points known as minutiae points [5].

Fingerprinting has been the most widely used during the $20^{\text {th }}$ century. The maturity of Biometric techniques and mainly the dramatic improvement of the captured devices have led to the proposal of fingerprinting in multiple applications but in the last years, minutiae have been the main type of algorithm used. The minutiae are relatively stable and robust to contrast, image resolution and global distortion as compared to other fingerprint representation. However, to extract minutiae from a poor quality image is not an easy task [6]. [7]. Proposed a local correlation based fingerprint matching algorithm. In the proposed algorithm the author used a window size of $42 \times 42$ pixels around the minutia locations in the template image and $32 \times 32$ pixels size windows around the corresponding location in the query image. The normalized cross-correlation between the query window and template window is computed and peak is detected. If the peak lie outside 10 pixels from the centre, the correlation between template and query window is zero otherwise this is the absolute value of correlation between the query and template window. The local correlation of all template windows with the corresponding regions in the query image are computed and mean correlation value is found. In this way all the possible correspondence from the alignment stage are tested and maximum correlation value over all the correspondence is taken as the matching score between the query and template image.

The aims of combining minutiae and correlation techniques are to improved robustness, completeness, usability, and efficiency.

\section{Research Methodology}

Fingerprint verification and identification systems follow minutiae-based approach. The Nigerian Immigration Service make use of AFIS which is minutia based, minutiae based algorithm has fixed radius and so can tolerate missing and spurious minutiae better than nearest neighbour based approach. Also minutiae are error tolerant too therefore makes coding a smooth one. However, minutia based technique are not noise tolerant as blurry images can be produced when noise interferes with it. This paper aims at trying to see how minutiae algorithm and correlation algorithm can be combined in a setting like the Nigerian Immigration for extraction and matching processes. For the purpose of this paper the mean of the score from the two approaches will be taken as the final score that would be compared with the established so as to increase FAR of an impostor before an acceptance or rejection is established.

\section{Implementation Process}

A growing security issue in Nigeria is treat and terrorism being on the hike side, research made it known that this detrimental acts and atrocities are being perpetrated by foreigners as a result of our porous borders. In view of this, this paper aims at trying to see how these acts can be reduced to the barest minimum if not totally eradicated; this paper encompasses the following implementation process:

The process of enrolment has all the enrollee's information and all necessary details that concern the enrolee and the biometric data captured in this stage and stored in the database for later use that is the process of verification and further update of information.

The process extraction and verification combines two approaches or techniques so as to obtain an efficient and prudent result in order to reduce or eradicate terrorism and high rate insecurity in Nigeria through the Nigerian Immigration Service using the proposed system.

4.1 Minutiae Feature Extraction from Image: this forms the part of the approach where the minutiae extraction is done and of course processes like binarization, thinning and bifurcation would be done in this part.

4.2 Rotation and Displacement of Image: This forms the part of the approach where the image is normalized to get an authentic and effective image to be stored in the database so as to aid the process of matching.

4.3 Template Database Storage: This part stores data that is, all the templates and information that are been generated from the process of minutiae extraction and rotation and displacement of image. 
4.4 The Enrollee Storage: The enrollee, this has all the details of all the people that have been enrolled and its stores them with the passport number. when there is need to view enrollee's details or make amends this can easily be done as the passport identification number can be used to trace individual's details and it makes the process of verification easier and faster as it saves time

4.5 The Verification Phase: This is where the verification is done, this is done by entering the enrollee's passport identification number and the enrollee is asked to take the verification process by placing his/her finger on the biometric fingerprint scanner (Secugen), it is captured and the admin verifies the live biometric fingerprint captured with the one in the database. Each approach (correlation techniques image matching and minutiae-based matching) computes its matching score and the mean of the score from the two approaches is compared with the established set threshold score and if the resulting score from the matching is greater than the threshold score the system accepts it and displays some information about the immigrant before the immigrant can be allowed into the country but if the resulting score is less than the set threshold the system ignores and moves to start so that the process can be taken again.

4.6 Enrollee: this describes the enrollee that is contains the enrollee's ID which is sub- divided into passport number which can take string characters, template 1 and 2 which can take binaries, images during capturing, extraction and verification which takes binaries

Biodata: This is the part that describes the biodata been stored in the database and it comprises of passport number which can take string characters, picture of the enrollee which can take binary characters, first name which can take alphabetic characters, last name which can take alphabetic characters as well Nationality of the enrollee which can also take alphabetic and string characters, date of birth which can take string characters date issued and expiry date can take string characters too.

4.6 Authentication: This is the part of the database that allows access into and out of the database, this part monitors the kind of people that uses the database and controls unwanted users and unnecessary logins and access into the database for more secured and protected database environment.

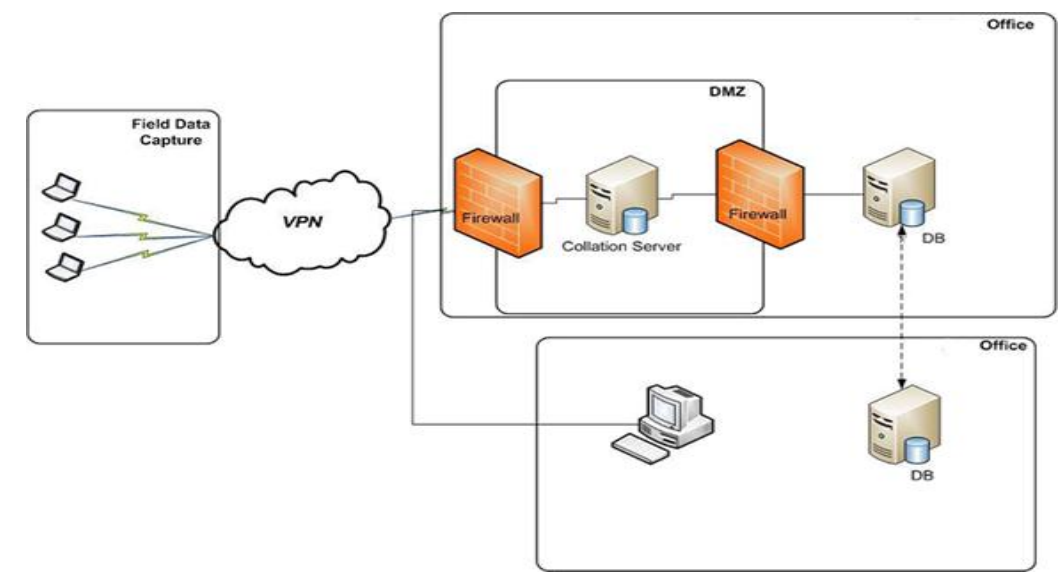

Architectural design of the system, Image source: Penop, (2012)

key

DMZ-Dimilitarized zone

Z9PE-D8- for more reliable network

VPN- this is called Virtual private Network

The above diagram shows the architectural design of the system

Filled Data Capture: This is where data collected from enrollees are been collected and stored in the database i.e extraction and matching process.

VPN: This is known as virtual private network that is a dedicated network for the system that protects the data been captured and stored in the database for present and future purposes.

Fire Walls: This is a kind of security that helps protect the network from spam ware and other attacks like hacking, virus attacks etc. of the proposed system.

Collation Server: This is responsible for dissemination of information to all clients on the network and even share of resources and other necessary things that are needed or used on the network. 
Z9PE-D8: This provides additional layer of security to the proposed system's local area network, it provides a good and secured motherboard for more reliable networking environment as it has in built dual Intel, Ethernet that leads to lower CPU utilization and yet it is very affordable

4.7 Template Matching: This part also consist of two main parts the minutiae matching part and the rotation and displacement of image matching part (correlation)

4.8 Minutiae Matching: Once the minutia feature extraction is done the next phase is to compare the live template with the stored template, the system fetches the template from the template database storage and compare it with the live template. Once the matching is done, the matching score from the two templates is computed.

\section{Conclusion}

This paper has been able to achieve some far-reaching objectives of dealing with illegal immigrants entering the country without valid documents and using some tactics and fraudulent acts to beat the machines put in place (fingerprint scanner) to check them while entering the country. By providing a reliable extraction and matching processes by combining two techniques namely, minutiae and correlation for the processes. The sole idea behind this is that one technique makes up for the deficiency of the other by so doing we obtain a reliable and efficient result and provide a more secured environment where immigrants will not be able to beat the system with their fraudulent tactics or act as it use to be before now. If this system is applied in the Nigerian Immigration settings, illegal immigrants will not be nabbed if they try to play pranks or find their way around beating the machine put in place to check immigrants coming into the country.

\section{References}

[1] Patric, Ebele and Chinedu, (2012). Terrorist activities and economic development in Nigeria: An early warning signal OIDA international Journal of sustainable development Vol 5, No.4, pp. 69-78, 2012.

[2] L. Hong, Y. Wan \& A. Jain, (1998). Fingerprint Image Enhancement: Algorithm and Performance Evaluation, IEEE Trans. Pattern Analysis and Machine Intelligence, vol. 20, no. 8, pp.777-789.

[3] S. Prabhakar, A.K. Jain, J. Wang, S. Pankanti, \& R. Bolle, (2000). Minutia Verification and Classification for Fingerprint Matching, In Proc. ICPR2000, 15th Int. Conf. Pattern Recognition, Barcelona, Spain.

[4] Frank Laczko, Abye Makonnen (2009). Migration in Nigeria: Thematic Document 2009 National Security and Migration: towards an Effective Cross-border management in Nigeria, (C) 2009 International Organization for Migration (IOM), International Organization for Migration, 17 route des Morillons 1211 Geneva 19, Switzerland.

[5] ICAO, (2006). "Machine readable travel documents", Technical report, ICAO 2006.

[6] B. Roli, S. Priti \& B. Punam, (2011). Minutia Extraction from Fingerprint Image: A review, International Journal of Computer Science Issues Vol. 8, Issue 5, No3, “001.

[7] K. Nandakumar and A. K. Jain, (2004). Local Correlation-based Fingerprint Matching, Proceedings of Indian Conference on Computer Vision, Graphics \& Image Processing, pp.1-6, Kolkata. 